Article

\title{
Role of the Nitric Oxide Reductase NorVW in the Survival and Virulence of Enterohaemorrhagic Escherichia coli during Infection
}

\author{
Marion Gardette ${ }^{1,2}$, Julien Daniel ${ }^{1}$, Estelle Loukiadis ${ }^{2,3}$ (I) and Grégory Jubelin ${ }^{1, *(D)}$ \\ 1 Université Clermont Auvergne, INRAE, MEDIS UMR454, F-63000 Clermont-Ferrand, France; \\ gardette.marion@gmail.com (M.G.); julien.daniel@inrae.fr (J.D.) \\ 2 Université de Lyon, CNRS, INRAE, Université Claude Bernard Lyon 1, VetAgro Sup, Laboratoire d'Ecologie \\ Microbienne, F-63280 Marcy l'Etoile, France; estelle.loukiadis@vetagro-sup.fr \\ 3 VetAgro Sup, Laboratoire national de référence des E. coli, F-63280 Marcy-l'Etoile, France \\ * Correspondence: gregory.jubelin@inrae.fr
}

Received: 24 July 2020; Accepted: 19 August 2020; Published: 21 August 2020

\begin{abstract}
Enterohaemorrhagic Escherichia coli (EHEC) are bacterial pathogens responsible for life-threatening diseases in humans, such as hemolytic and uremic syndrome. It has been previously demonstrated that the interplay between EHEC and nitric oxide (NO), a mediator of the host immune innate response, is critical for infection outcome, since NO affects both Shiga toxin (Stx) production and adhesion to enterocytes. In this study, we investigated the role of the NO reductase NorVW in the virulence and fitness of two EHEC strains in a murine model of infection. We determined that the deletion of norVW in the strain O91:H21 B2F1 has no impact on its virulence, whereas it reduces the ability of the strain O157:H7 620 to persist in the mouse gut and to produce Stx. We also revealed that the fitness defect of strain $620 \triangle n o r V W$ is strongly attenuated when mice are treated with an NO synthase inhibitor. Altogether, these results demonstrate that the NO reductase NorVW participates in EHEC resistance against NO produced by the host and promotes virulence through the modulation of Stx synthesis. The contribution of NorVW in the EHEC infectious process is, however, strain-dependent and suggests that the EHEC response to nitrosative stress is complex and multifactorial.
\end{abstract}

Keywords: EHEC; Shiga toxin; virulence; nitric oxide; NO reductase

\section{Introduction}

Enterohaemorrhagic Escherichia coli (EHEC) are foodborne pathogens responsible for intestinal disorders in humans that can evolve into life-threatening diseases, such as hemorrhagic colitis and hemolytic-uremic syndrome (HUS) [1-3]. EHEC are classified by O:H serotype based on their lipopolysaccharide and flagellar antigens. Among the limited number of serotypes frequently involved in human infections [4], O157:H7 is recognized as the major serotype involved in EHEC outbreaks and sporadic cases of infection worldwide [5]. However, the incidence of non-O157:H7 strains has steadily increased these last years, and serotypes such as O26:H11, O103:H2, O91:H21, and O80:H2 are now involved in a substantial proportion of infection cases [6,7]. The cardinal virulence factor in EHEC is Shiga toxins (Stx), which are $A B_{5}$ proteins where the A subunit is the catalytic protein and the five $B$ subunits are required for binding at the surface of target cells [8]. Once produced in the gut, Stx crosses the intestinal epithelial barrier and gains access to the systemic circulation, where it targets the glycolipid globotriaosylceramide-3 (Gb3) receptors of endothelial cells [9]. The internalization of Stx alters the ribosomal function and induces the necrosis or apoptosis of vascular endothelial cells, leading to symptoms such as hemorrhagic colitis and HUS [10]. EHEC can produce two distinct forms of 
Stx-Stx1 and Stx2-alone or in combination, and several subtypes have been described for each type of Stx [11]. The stx 1 and st $x 2$ genes are located within lambdoid phages integrated within the bacterial chromosome, and their expression is mainly activated through the induction of the SOS response [12]. Epidemiological studies have shown that EHEC strains producing Stx2, especially subtypes Stx2a and Stx2d, are more commonly associated with serious human diseases such as HUS than Stx1-producing strains $[13,14]$.

Although critical for EHEC virulence, Stx is not sufficient per se to cause disease in humans, and many Shiga-toxin-producing E. coli (STEC) strains have never been associated with cases of infection [4]. The virulence potential of EHEC strains results from the association of $s t x$ genes with other virulence or fitness factors encoding genes located on the chromosome or on mobile elements such as plasmids. Among these factors, the Locus of Enterocyte Effacement (LEE) is frequently detected in EHEC strains, causing severe infections. LEE genes encode the type III secretion system (T3SS) which is involved in the formation of attaching and effacing (A/E) lesions on infected epithelial cells, leading to an intimate attachment of EHEC to the gut mucosa [15]. The norV gene was also identified as a putative virulence determinant in the EHEC O157:H7 strain TW14359, which has been involved in an outbreak with a particularly high rate of HUS [16,17]. nor V and the co-transcribed norW gene encode a flavorubredoxin and a NADH:(flavo)rubredoxin reductase, respectively, that are involved in nitric oxide (NO) detoxification under anaerobic and microaerobic growth conditions [18-20]. NO is a highly reactive inorganic free radical with pleiotropic functions in mammals and has notably antimicrobial properties that make it efficient in fighting against incoming pathogens as part of the host immune response [21]. In the case of EHEC, the interplay between NO and the pathogen appears to be even more critical for the outcome of infection, since NO has been shown to modulate the expression of both the stx and LEE genes [22-25]. In addition, NorV has been shown to promote EHEC survival and Stx2 production within macrophages, suggesting that NorV contributes to EHEC virulence [26].

In this context, the aim of this work was to evaluate the role of nor $V$ in the pathogenicity of two EHEC strains of serotype O157:H7 or O91:H21, using mice for an animal model of infection. We determined that the deletion of norVW highly affects the efficiency of the strain O157:H7 to colonize the gut and release Stx in vivo. In contrast, norVW appears to be dispensable for the virulence of the O91:H21 strain. We also demonstrated that norVW is critical for the efficient fitness of the O157:H7 strain in the gut of infected mice. Importantly, the advantage procured by the presence of nor $V W$ is significantly decreased when NO production by the host is inhibited, strongly suggesting that NorV is involved in NO detoxification during the infectious process. Altogether, our work highlights the role of the NO reductase NorV in the fitness and virulence of some, but not all, EHEC strains during the infectious process.

\section{Results}

\subsection{Selection of nor $V^{+}$EHEC Strains}

Two forms of the nor $V$ gene exist in EHEC, an intact and functional gene $\left(n o r V^{+}\right)$and an inactive gene with a $204 \mathrm{bp}$ deletion $\left(\right.$ nor $\left.V^{-}\right)[19,26]$. We determined the norV status in 34 EHEC strains belonging to different serotypes frequently involved in human infection (Table 1). Three of the six tested O157:H7 isolates and all of the non-O157 strains possess an intact nor $V$ gene. No correlation was observed between the nor $V$ status and the presence of virulence genes eae (encoding intimin), stx1, and stx2. These data are consistent with previous results, indicating that the truncated form of the norV gene is strictly detected in the O157:H7 strains [19,26]. Three O157:H7 and two non-O157:H7 strains carrying the nor $V^{+}$gene were next selected for further investigation. For the O157:H7 serotype, the selected strains were (i) FCH6, a typical O157 strain producing the Stx2a variant and involved in an outbreak ([27] and personal communication with Sergentet D. from [28]); (ii) 620, a sorbitol-fermenting clone producing Stx2a and associated with a high incidence of HUS and high fatality rate (personal communication with Loukiadis E. from [29]); (iii) 540, a Stx1 and Stx2a-producing strain which has been 
involved in sporadic cases of infection ([30] and personal communication with Loukiadis E. from [29]). The three O157:H7 strains all possess the LEE operon. The two selected non-O157:H7 strains were 21765, an $\mathrm{LEE}^{+}$and Stx2a+ O26:H11 strain [31], and B2F1, a LEE ${ }^{-}$O91:H21 strain producing two Stx2d variants [32]. Both isolates were recovered from HUS cases [33,34].

Table 1. Distribution of norV, eae, and stx genes in 34 enterohaemorrhagic Escherichia coli (EHEC) strains ${ }^{a}$.

\begin{tabular}{|c|c|c|c|c|c|}
\hline Serotype & Strain & norV & $e a e^{\mathbf{b}}$ & stx 1 & $s t x 2$ \\
\hline \multirow{6}{*}{ O157:H7 } & 620 & + & + & - & + \\
\hline & 540 & + & + & + & + \\
\hline & FCH6 & + & + & - & + \\
\hline & RD9 & - & + & + & + \\
\hline & EDL 933 & - & + & + & + \\
\hline & Sakaï & - & + & + & + \\
\hline \multirow{4}{*}{ O26:H11 } & 21765 & + & + & - & + \\
\hline & 37.40 & + & + & + & - \\
\hline & $279 / 8$ & + & + & + & - \\
\hline & 11368 & + & + & + & - \\
\hline \multirow{5}{*}{ O103:H2 } & PMK5 & + & + & + & - \\
\hline & 590 & + & + & + & - \\
\hline & 2503 & + & + & + & - \\
\hline & 03.35 & + & + & + & - \\
\hline & 2455-1 & + & + & + & + \\
\hline \multirow{3}{*}{ O111:H8 } & CL37 & + & + & - & - \\
\hline & $\mathrm{J} 43$ & + & + & + & - \\
\hline & $622-4$ & + & + & + & + \\
\hline \multirow{3}{*}{ O145:H28 } & 2513-21 & + & + & - & + \\
\hline & 991 & + & + & + & - \\
\hline & 1036 & + & + & - & + \\
\hline \multirow{3}{*}{ O121:H19 } & 12652 & + & + & - & + \\
\hline & 12805 & + & + & - & + \\
\hline & S3075 & + & + & - & + \\
\hline $\mathrm{O} 45: \mathrm{H} 2$ & 12047 & + & + & + & - \\
\hline \multirow{3}{*}{ O91:H21 } & 13199 & + & - & + & + \\
\hline & B2F1 & + & - & - & + \\
\hline & 13694 & + & - & - & + \\
\hline \multirow{2}{*}{ O113:H21 } & 13341 & + & - & - & + \\
\hline & 14032 & + & - & - & + \\
\hline O113:H4 & 13137 & + & - & + & - \\
\hline \multirow{3}{*}{ O80:H2 } & 38009 & + & + & - & + \\
\hline & RDEx444 & + & + & - & + \\
\hline & 40963 & + & + & - & + \\
\hline
\end{tabular}

a The symbols + and - indicate, respectively, the presence and the absence of indicated genes except for nor $V$, for which the symbol - indicates the presence of a truncated form of the gene. ${ }^{b}$ eae encodes intimin and is a marker of the presence of the locus of enterocyte effacement (LEE).

\subsection{Virulence of nor $V^{+}$EHEC Strains in a Mouse Model of Infection}

We first determined the virulence potential of the selected strains using a mouse model of infection (see M\&M). Streptomycin-treated mice were orally infected with a streptomycin-resistant variant of each strain (hereafter named wild-type (WT) strains for more convenience), as well as with the reference strain EDL933 (an O157:H7 strain with a truncated form of norV), and body weight was recorded daily to evaluate the severity of infection (Figure 1a). Mice infected with 540, FCH6, or 21,765 
strains gained body weight during the course of infection, similarly to uninfected mice. Infection with EDL933 and 620 led to the same body weight pattern, characterized by a regular increase during the first 6 days and a slight but significant decrease at day 7 post-infection.

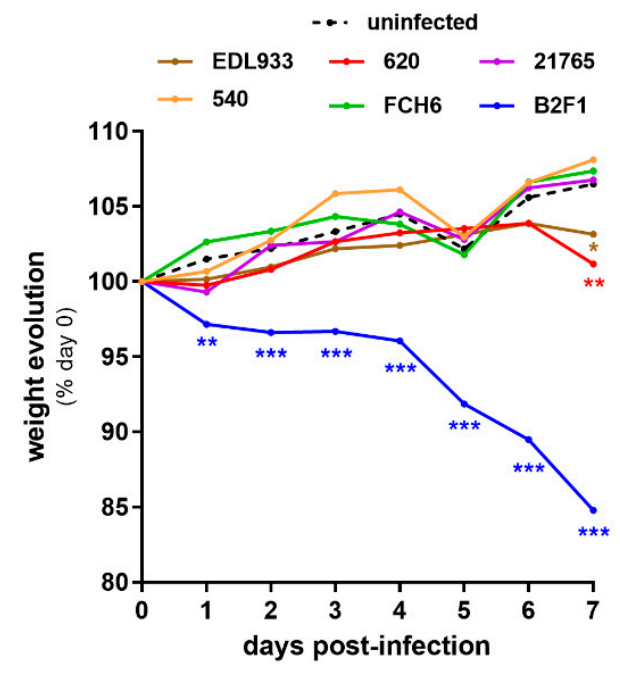

(a)

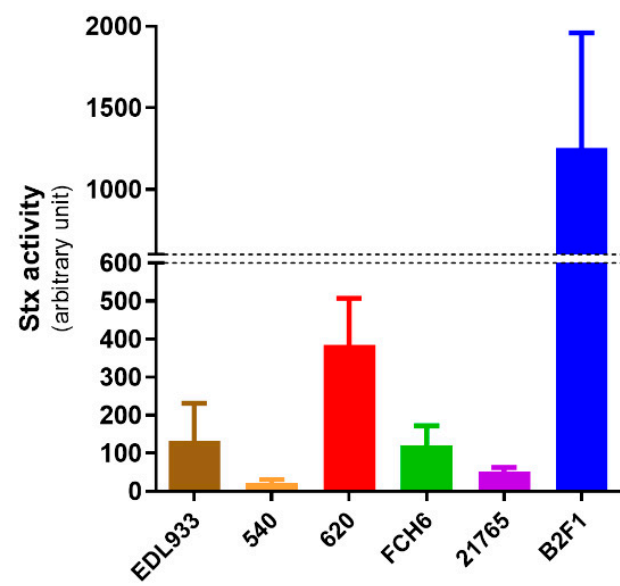

(b)

Figure 1. Virulence potential of EHEC strains in mice. (a) Groups of 5 to 20 mice were infected by indicated strains at day 0 , and the mouse weights were determined daily over a 7-day period. Data are presented as the percentage relative to animal weight at the day of infection, (day 0 ) and curves represent mean values. A multiple two-tailed unpaired t-test was applied to compare each infected group with the uninfected group every day. ${ }^{*} p<0.05,{ }^{* *} p<0.01,{ }^{* * *} p<0.001$. (b) At day 3 post-infection, the Stx activity of fecal samples was quantified using the Vero-d2EGFP cell line. Results are presented as means and standard deviations, with at least 4 mice per group.

In contrast, the B2F1-infected mice lost weight as soon as day 1 post-infection, and their body weight was significantly lower than that of uninfected mice during the entire course of infection. In addition, the B2F1-infected mice developed serious clinical signs of disease, as previously described [35], and $30 \%$ of the mice were moribund or died during the experiment (Figure S1). We also evaluated the amount of released Stx at day 3 post-infection through the quantification of the Stx activity from fecal samples using Vero-d2GFP, a green fluorescent protein (GFP)-producing cell line used to monitor protein synthesis inhibition [36]. These data revealed that B2F1 and 620 present the highest level of Stx activity in vivo among the tested strains (Figure 1b), correlating well with the degree of weight loss and symptoms observed in the infected mice. B2F1 and 620 strains were then used to investigate the role of the norVW operon in EHEC virulence and fitness.

\subsection{Role of the norVW Operon in EHEC Virulence}

To assess the contribution of norVW to EHEC virulence, we orally inoculated mice with WT or $\triangle$ norVW mutant from 620 or B2F1 strains and evaluated the infection outcome by analyzing the weight loss and clinical signs of the animals. The body weight curves were not significantly different between the WT and $\triangle$ norVW strains both for 620 and B2F1 (Figure 2a). Indeed, the mice infected with $620 \mathrm{WT}$ or $\Delta$ nor $V W$ showed a weight increase during the first 6 days post-infection, whereas the mice challenged with B2F1 WT or $\triangle$ norVW both exhibited serious clinical symptoms and considerable weight loss. 


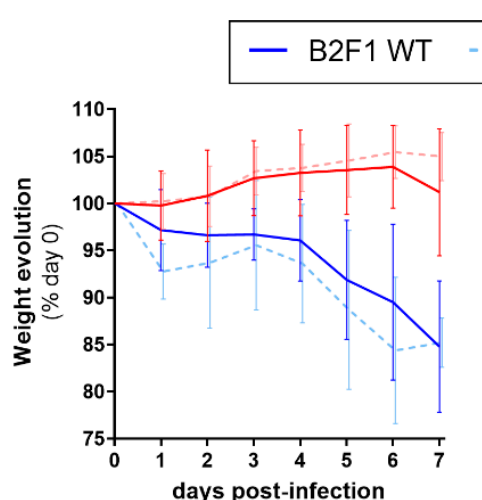

(a)

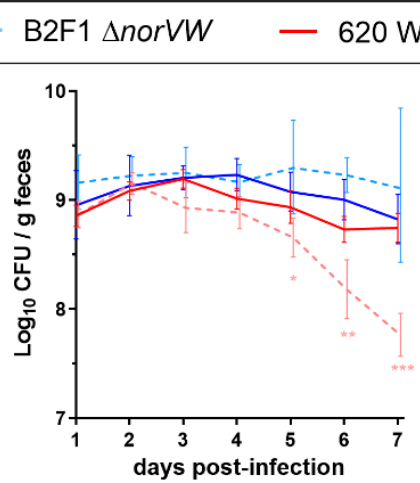

(b)

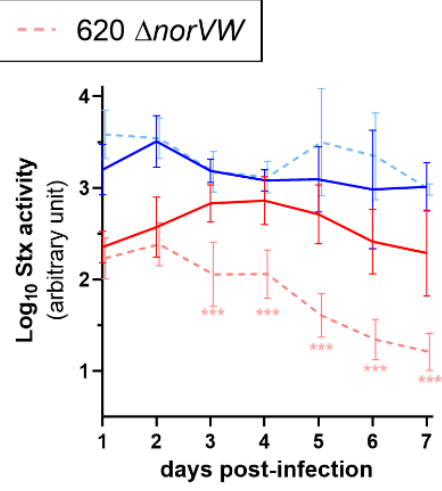

(c)

Figure 2. Deletion of the norVW operon differentially affects the virulence of strains 620 and B2F1. Groups of 10 to 20 mice were infected with WT or $\Delta$ norVW mutant from the strains 620 or B2F1. (a) Mouse weights were determined daily following infection, and body weight curves are presented as the percentage relative to animal weight at the day of infection (day 0). Curves represent mean values with standard deviation (SD). (b) At the indicated time points, EHEC shedding was quantified by plating fecal samples on LB + Sm plates. Curves represent mean values with SD. (c) Stx activity from fecal samples was quantified using the Vero-d2EGFP cells. Curves represent mean values with SD. A multiple two-tailed unpaired t-test was performed to compare the WT and $\Delta$ nor $V W$ groups of mice infected with 620 or B2F1 every day. ${ }^{*} p<0.05,{ }^{* *} p<0.01,{ }^{* * *} p<0.001$.

We next evaluated the consequence of nor $V W$ deletion on the ability of strains to persist in the gut. In mice infected with B2F1, the fecal shedding was stable during the course of infection, both for the WT and $\Delta$ norVW strains (Figure 2b). In contrast, significantly fewer CFU were recovered in the feces of mice infected with $620 \Delta n o r V W$ than from those infected with 620 WT from day 5 to day 7 post-infection (Figure 2b). This result suggests that norVW contributes to the persistence of strain 620 in the gut lumen. To assess whether norVW deletion also affects the ability of EHEC to adhere to the gut epithelium in vivo, adherent bacteria were quantified from colonic tissues at day 7 post-infection (Figure S2). The number of adherent EHEC was highly variable between the mice, and we were not able to detect significant differences between the WT and $\Delta$ norVW strains from both the 620 and B2F1 isolates. We next investigated whether nor $V W$ deletion affects the activity of Stx in vivo via the quantification of Stx activity from animal feces (Figure 2c). First, we noticed that the Stx activities recorded from the B2F1-infected mice were higher than those recorded from animals infected with 620 during the entire course of infection, correlating well with the severity of symptoms observed for these mice. We next compared the Stx activities between the WT and $\Delta$ norVW mutant for each strain. We did not observe differences between the mice infected with B2F1 WT or mutant strains, demonstrating that the norVW operon did not alter the Stx activity in B2F1. In contrast, the Stx activities recorded from $620 \Delta$ nor $V W$-infected mice were significantly lower than the Stx activities measured from mice infected with the WT strain between day 3 and day 7 post-infection. This data reveals that the deletion of the nor $V W$ operon in 620 negatively affects the level of Stx activity in infected animals. Altogether, these data demonstrate that the virulence of the $\Delta$ nor $V W$ mutant is attenuated for strain 620 , but not for strain B2F1, and highlight that the expression of nor $V W$ can influence the virulence of some, but not all, EHEC strains.

\subsection{Role of the norVW Operon in the Fitness of EHEC during Mouse Infection and Influence of NO Synthase Activity from the Host}

To assess the contribution of norVW in the fitness of strains 620 and B2F1 in the gut, the mice were co-infected with an equal ratio of WT and $\Delta$ norVW strains, and both populations were monitored over the course of infection to determine the competitive indices (ratio WT/mutant) (Figure 3a). 


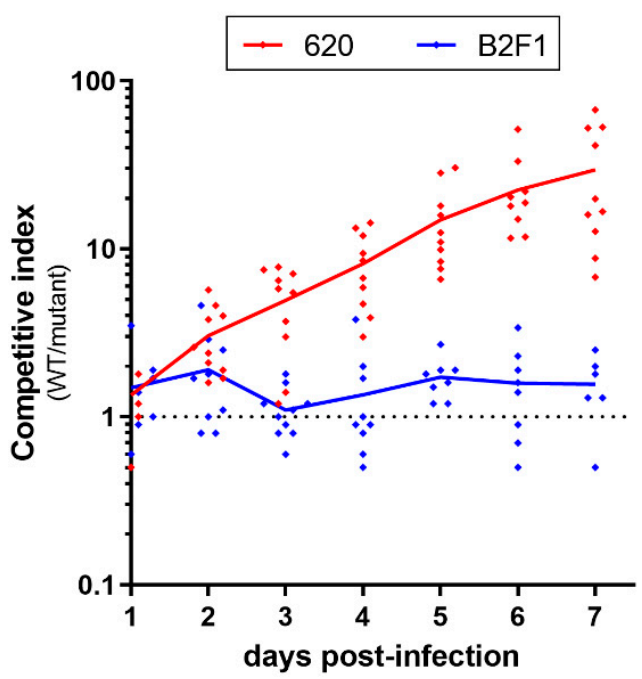

(a)

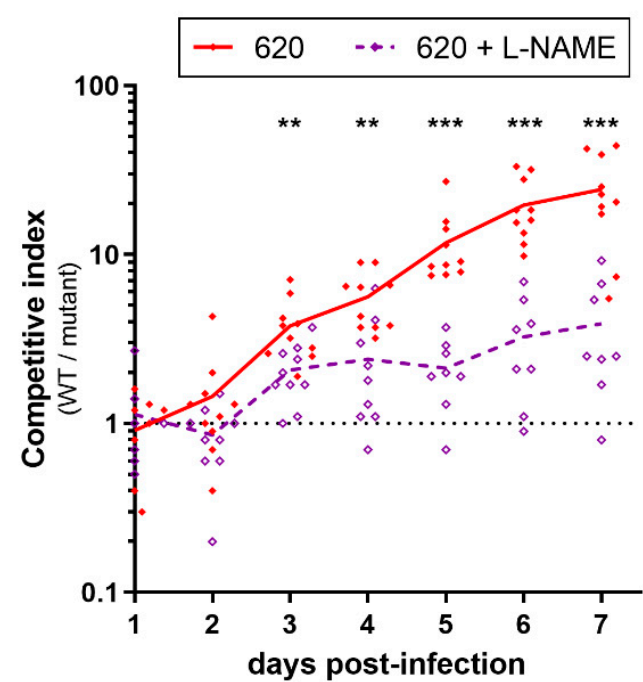

(b)

Figure 3. Role of the norVW operon in EHEC fitness during the infection and influence of NO synthase activity from the host. (a) Mice were co-infected with an equal mixture of WT and $\Delta$ norVW mutant from strains 620 or B2F1. At the indicated time points, feces were sampled and spotted on LB $+\mathrm{Sm}$ plates and $\mathrm{LB}+\mathrm{Sm}+$ Kan plates to count, respectively, the WT $+\Delta$ nor $V W$ mutants and $\Delta$ norVW mutants alone. The WT population was obtained by subtracting the $\Delta$ nor $V W$ mutant colony-forming unit (CFU) from the total EHEC CFU. Competitive indices (ratio WT/mutant) were calculated for each animal. Each dot represents one mouse, and curves represent mean values. (b) A similar competition assay was performed using the $620 \mathrm{WT}$ and $\Delta$ norVW strains, and mice from one group were treated with the NOS inhibitor L-NAME throughout the experiment and mice from the other group were left untreated. A multiple two-tailed unpaired t-test was performed to compare both groups every day. ${ }^{* *}$ $p<0.01 ;{ }^{* * *} p<0.001$.

The deletion of genes nor VW had no impact on the B2F1 colonization efficiency, since the calculated competitive indices remained close to 1 throughout the experiment. In contrast, the competitive indices greatly increased for 620 and reached a mean value of 30 at day 7 post-infection, indicating that 620 WT outcompetes the $\triangle n o r V W$ mutant in vivo. These data demonstrate that the norVW operon contributes to the fitness of strain 620 in the mouse gut but does not influence the behavior of strain B2F1. Because NO is released in the mouse gut during EHEC infection [25], we wondered if the fitness defect observed with the $620 \Delta n o r V W$ strain is related to the NO production by the host during infection. The mice were then co-infected with the $620 \mathrm{WT}$ and $\Delta$ norVW strains and treated or not with L-NAME, a specific inhibitor of NO synthase (NOS) activity (Figure 3b). The competitive indices calculated from the mice treated with L-NAME stayed very low (maximal mean value of 3.9 at day 7 post-infection), and were significantly lower than those obtained from the untreated mice. These differences were observed as soon as day 3 post-infection and increased over time until the end of the experiment. These data demonstrate that the competitive advantage of the WT strain over the $\Delta$ norVW mutant in the mouse gut is due, at least partially, to NO production by the host. Finally, we also evaluated the role of NorVW in the fitness of the three other EHEC strains initially selected (540, FCH6, and 21765) by co-infection experiments (Figure 4). For the three strains, the mutant was outcompeted by the WT strain by a factor ranging from 19 to 1400 at day 7 post-infection. These data indicate that NorVW also participates in the fitness of other $\mathrm{O} 157$ and non-O157 strains during mouse infection, as initially determined with the strain 620. 


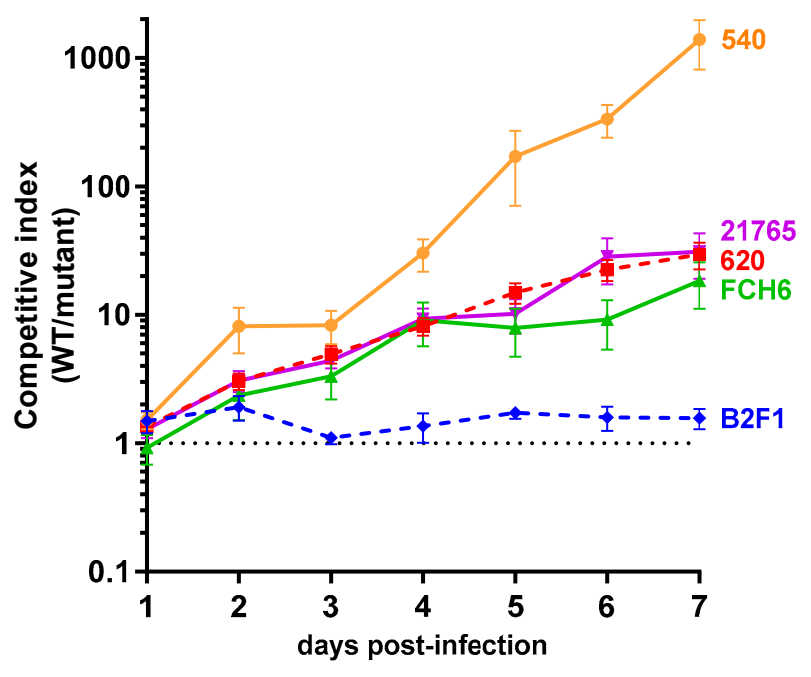

Figure 4. Role of the nor VW operon in the fitness of other EHEC strains during infection. Groups of 10 mice were co-infected with an equal mixture of WT and $\Delta$ norVW mutant from strains FCH6, 540, and 21,765 (and 620 and B2F1 as references). At different time points, feces were sampled and spotted on $\mathrm{LB}+\mathrm{Sm}$ plates and LB $+\mathrm{Sm}+$ Kan plates to count, respectively, the $\mathrm{WT}+\Delta$ norVW mutants and $\Delta$ norVW mutants alone. The WT population was obtained by subtracting the $\Delta$ norVW mutant CFU from the total EHEC CFU. Competitive indices (ratio WT/mutant) were calculated for each animal. Curves represent the mean values with standard errors of the mean.

\section{Discussion}

During the past decades, NO has been recognized as an important player in the immune system, participating notably to host defense against infectious agents [21]. In the case of EHEC infection, several works also demonstrated that the pathogen is able to sense NO in the gut and to adapt its gene expression accordingly, making the interplay between $\mathrm{NO}$ and EHEC critical for the infection outcome [22,25,37-39]. Among the NO-regulated factors, the NO reductase NorVW was identified as a putative virulence determinant in the EHEC O157:H7 strain TW14359 and was shown to promote EHEC survival within macrophages $[16,26]$. NorV was also identified as one of the immunogenic antigens expressed by EHEC within the gastrointestinal tract of HUS patients [38]. The aim of this study was to examine the contribution of the NO reductase NorVW to EHEC pathogenesis. Using a mouse model of infection, we demonstrated that the deletion of the gene norVW impacts the virulence of strain O157:H7 620, but not of strain O91:H21 B2F1, suggesting that the activity of the NO reductase is important in the infectious process of some, but not all, EHEC strains. In addition, the fitness of four of the five tested EHEC strains was affected in the gut of infected mice in the absence of the norVW operon. The altered fitness of $620 \Delta n o r V W$ was furthermore attenuated in L-NAME-treated mice, producing a lower amount of NO. In this condition, the fitness of the WT and $\Delta n o r V W$ strains was nearly equivalent, and this data strongly suggests that NorVW plays a role in the detoxification of NO produced by the host in response to infection [25]. In addition to NorVW, E. coli harbor three other enzymes known to detoxify NO: the flavohaemoglobin Hmp, which oxidizes NO to nitrate or reduces NO to nitrous oxide $[19,40]$; the periplasmic nitrite reductase NrfA, which transforms NO into ammonia [41]; and the hybrid cluster protein Hcp, which catalyzes the reduction of NO to nitrous oxide [42]. In bacterial cells, the quantitative contribution of Hmp, NrfA, Hcp, and NorV to $\mathrm{NO}$ detoxification varies significantly depending on the $\mathrm{NO}$ and dioxygen concentrations, since both molecules affect the synthesis and/or activity levels of these enzymes [43-45]. However, the variations in the cooperativity level of NO-detoxifying enzymes have never been examined between $E$. coli strains. Whereas genes $h m p, n r f A, h c p$, and norVW belong to the core genome of E. coli, it is conceivable that their expression level and/or enzyme efficiency differ between strains 620 and B2F1, explaining why NorVW is important for the fitness and virulence of 620 but not of B2F1. Otherwise, B2F1 may also 
possess other unidentified NO scavenging mechanisms, as was shown in the uropathogenic E. coli strain CFT073 [46].

One important feature observed in our study is that norVW deletion leads to a significant decrease in the Stx activity level in the gut of mice infected by 620, but not of mice infected by B2F1. It should be noticed that 620 and B2F1 produce distinct variants of Stx2 (Stx2a for 620 and two Stx2d for B2F1), and it is conceivable that their regulation may differ. In the reference strain EDL933, which produces the Stx2a variant like 620, NO modulates the st $x$ gene expression in a RecA-dependant manner by affecting the induction level of the phage lytic cycle [39]. Interestingly, it has been shown that the regulation of the two st $2 \mathrm{~d}$ operons in B2F1 is different, and only one st $x$ allele has an expression linked to its bacteriophage induction [32], leaving open the possibility that the RecA-dependent effect of NO may not occur for one of the stx alleles. The reduction in the Stx activity observed in mice infected by $620 \Delta$ nor $V W$ occurred as soon as day 3 post-infection and did not result from a reduced level of gut colonization by the mutant, which was not observed before day 5 post-infection (compare Figure $2 b, c)$. Actually, the reduced colonization ability of $620 \Delta n o r V W$ could even be a consequence of the decreased Stx activity, since it was previously reported that Stx may enhance the capacity of EHEC O157:H7 to adhere to epithelial cells and to colonize the mouse intestine $[47,48]$. The low Stx activity recorded from mice infected with the $\triangle$ nor $V W$ mutant probably results from an increase in the NO concentration in the absence of NO reductase, and suggests that NO represses Stx production. The influence of NO on Stx synthesis has been well demonstrated in several studies, but appears to be highly variable. Indeed, NO was shown in vitro to inhibit Stx production during standard LB culture [39], whereas it activates Stx synthesis under anaerobic conditions [24]. The infection of macrophages with nor $V^{+}$EHEC strains reduces the NO concentration and leads to a higher level of St 2 than within macrophages infected with norV mutants [26]. Our team also recently demonstrated that the treatment of EHEC-infected mice with a NO synthase inhibitor significantly decreases the Stx activity in the gut [25]. Altogether, these data reveal that the modulation of Stx synthesis by NO (and indirectly by the activity of NO detoxification systems) is highly complex and sensitive, and affected by multiple environmental conditions.

The role of NO detoxification systems in virulence has been highlighted in vivo for other bacterial pathogens. If numerous studies have demonstrated the role of Hmp in the virulence of Salmonella enterica, Vibrio cholera, Staphylococcus aureus, Yersinia pestis, and uropathogenic E. coli [49-54], very few data exist for the less studied NO detoxification systems. In the case of NorVW, it has been shown that gene deletion affects the virulence of Pseudomonas aeruginosa in a silkworm model [55] and the virulence of Aeromonas hydrophila in Zebrafish, a bacterium responsible for diseases in amphibians, fish, and reptiles [56]. In contrast, norV deletion in S. enterica did not affect the virulence in mice [49,57]. To our knowledge, the contribution of NorV in the infectious process of intestinal or extra-intestinal pathogenic E. coli has never been documented, and this study is the first to demonstrate a role of the NO reductase NorV in the virulence of $E$. coli. Intriguingly, the occurrence of a deletion event in nor $V$ gene during the phylogenetic evolution of $\mathrm{O} 157$ is contradictory with the role of NorV in EHEC virulence. The truncated form of nor $V$ is, however, essentially detected in Stx $1^{+}$St $x 2^{+}$EHEC strains and very rarely in EHEC strains, producing only Stx2 [58]. Because the latter group is more commonly associated with the development of severe symptoms in infected patients $[59,60]$ and the molecular reasons for this are still unknown, it is tempting to speculate that the presence of a functional NO reductase NorV in these strains can contribute to their high potential for virulence. Further investigations are required to understand and characterize the contribution of NorV and other NO detoxification systems in the virulence of EHEC, potentially leading to their exploitation as new targets for antibacterial agents.

\section{Materials and Methods}

\subsection{Bacterial Strains, Growth Conditions, and Construction of Mutants}

The bacterial strains and plasmids used in this study are listed in Table S1, and the primer sequences are listed in Table S2. The status of the norV gene was determined in each EHEC strain 
either by DNA sequence analysis if the genome was publicly available or by PCR using the primers norV ${ }^{+}-\mathrm{F}$ and norV ${ }^{+}-\mathrm{R}$. For selected strains $(620, \mathrm{FCH} 6,540,21,765$, and B2F1), a streptomycin-resistant variant was constructed by replacing the rspL gene with gene rpsL150 amplified from MC4100 (confers resistance to streptomycin), as described in [61]. The deletion of the norVW operon was performed in EHEC strains using the one-step PCR-based method [62] using the primers norVW-mut-F and norVW-mut-R. Chromosomal deletions were confirmed by PCR using the primers norVW-verif-F and norVW-verif-R, followed by DNA sequencing. Bacteria were routinely grown in Luria-Bertani (LB) medium (Sigma-Aldrich, St Quentin Fallavier, France) at $37^{\circ} \mathrm{C}$, unless otherwise indicated. When required, antibiotics were used at the following concentrations: ampicillin (Amp), $50 \mu \mathrm{g} \mathrm{mL}{ }^{-1}$; kanamycin (Kan), $25 \mu \mathrm{g} \mathrm{mL}{ }^{-1}$; atreptomycin $(\mathrm{Sm}), 50 \mu \mathrm{g} \mathrm{mL} \mathrm{L}^{-1}$.

\subsection{Mouse Infection}

C57BL/6 mice with specific-pathogen-free (SPF) status were purchased from Janvier Labs (Le-Genest-St-Isle, France). Female mice aged 5 weeks were used throughout the experiments. They were housed in cages containing no more than five animals, maintained under a $12 \mathrm{~h}$ light/dark cycle at a temperature of $21 \pm 2{ }^{\circ} \mathrm{C}$, and fed with standard diet and water ad libitum. The experiments performed herein were reviewed and approved by the Auvergne Committee for Animal Experimentation C2EA (Agreement $N^{\circ}$ 7289-2016093010075533). Mouse experiments were performed with 5-20 mice per group. The mice were given drinking water containing $5 \mathrm{~g} / \mathrm{L}$ of streptomycin sulfate (Sigma-Aldrich, St Quentin Fallavier, France) one day prior to infection and during the 7 days following infection. For some groups, drinking water was also supplemented with $1 \mathrm{~g} / \mathrm{L}$ of the NOS inhibitor $\mathrm{N}^{\omega}$-nitro-L-arginine methyl ester hydrochloride (L-NAME; Enzo Life Science, Villeurbanne, France), and changed daily over the course of the experiment. The mice were infected intragastrically at day 0 with $100 \mu \mathrm{L}$ of PBS (Sigma-Aldrich, St Quentin Fallavier, France) containing $10^{7}$ bacteria. For this, the bacteria were grown in LB to the mid-logarithmic phase, collected by centrifugation, and resuspended to a concentration of $10^{8} \mathrm{CFU} / \mathrm{mL}$ in sterile phosphate-buffered saline (PBS). Uninfected mice were given PBS only. The body weight and clinical signs of mice were monitored daily to evaluate the severity of infection. Mice presenting a weight loss $>15 \%$ compared to their body weight at day 0 , or presenting severe clinical symptoms such as ataxia and lethargy, were immediately euthanized. Fecal samples were collected every day following infection. Feces were homogenized in PBS and diluted before plating on LB + $\mathrm{Sm}$. Resuspended feces were centrifuged, and the supernatant was filtered and stored at $-80{ }^{\circ} \mathrm{C}$ for subsequent Stx activity quantification. The mice were euthanized at day 7 post-infection. At necropsy, a piece of colon was collected, cut longitudinally, and washed extensively in PBS. Crushing in PBS, dilution, and plating were performed to quantify the bacteria adhering to the gut mucosa.

For the co-infection experiments, the mice were infected with a mix containing $10^{7}$ each of the WT and $\Delta n o r V W$ strains. The bacteria were enumerated in feces during the 7 days post-infection. Fecal samples dilutions were plated on $\mathrm{LB}+\mathrm{Sm}$ or $\mathrm{LB}+\mathrm{Sm}+\mathrm{Km}$ agar plates to count the WT $+\Delta n o r V W$ strains and $\triangle n o r V W$ alone, respectively. Competitive indices were calculated daily by dividing the output ratio (WT/mutant) by the corresponding input ratio (WT/mutant from mouse inoculum).

\subsection{Stx activity Quantification}

The Shiga toxin activity in the fecal samples was measured using a Vero-d2EGFP cell line that harbors a destabilized variant (half - life $=2 \mathrm{~h}$ ) of enhanced green fluorescent protein (EGFP), as described previously [36,63]. Purified Stx2 (Toxin Technology, Sarasota, FL, USA) was used as an internal standard. Three days prior to intoxication, the Vero-d2EGFP cells were seeded in black 96-well plates with a clear bottom at $3 \times 10^{4}$ cells per well. They were grown in $5 \% \mathrm{CO}_{2}$ at $37{ }^{\circ} \mathrm{C}$ under humidified conditions in a complete medium made of Annonces

Dulbecco's modified Eagle's medium (DMEM) supplemented with 10\% fetal bovine serum (Gibco, Fisher Scientific, Illkirch, France), Zell Shield (Minerva Biolabs, Berlin, Germany), and $200 \mu \mathrm{g} \mathrm{mL}^{-1} \mathrm{of}$ Geneticin (Gibco, Fisher Scientific, Illkirch, France). The samples to be tested as well as purified with 
Stx2 were diluted in complete medium, transferred to Vero-d2EGFP-containing plates, and incubated at $37^{\circ} \mathrm{C}$ for $16 \mathrm{~h}$ in a $5 \% \mathrm{CO}_{2}$ humidified incubator. After incubation, the samples were removed and $100 \mu \mathrm{L}$ of PBS was added to each well. The EGFP fluorescence from the Vero-d2EGFP was quantified in a Spark microplate reader (Tecan, Lyon, France) with an excitation at $485 \pm 20 \mathrm{~nm}$ and emission at $530 \pm 20 \mathrm{~nm}$. The Stx activity was expressed as an arbitrary unit by comparing the fluorescence values from the standard curve obtained with purified Stx2.

\subsection{Statistical Analyses}

All the statistical analyses were performed using the Prism software version 8 (GraphPad Software, San Diego, California, USA). Each dataset was analyzed by the ROUT method to exclude outliers with a $Q$ value of 1\%. An unpaired two-tailed Student's $t$ test was used to determine significant differences between the two groups. $p<0.05$ was considered significant.

Supplementary Materials: The following are available online at http://www.mdpi.com/2076-0817/9/9/683/s1. Figure S1. Survival rate of mice infected with EHEC strains. Figure S2. Deletion of the norVW operon does not affect EHEC adhesion to colonic mucosa. Table S1. Bacterial strains and plasmids used in this study. Table S2. Primers used in this study. References [64-70] are cited in the Supplementary Materials.

Author Contributions: Conceptualization, M.G., E.L. and G.J.; Investigation, M.G., J.D. and G.J.; Writing一original draft, M.G., E.L. and G.J. All authors have read and agreed to the published version of the manuscript.

Funding: This work was supported by funding from INRAE and VetAgro Sup institutes. Marion Gardette was a $\mathrm{PhD}$ Research Fellow funded by INRAE and VetAgro Sup institutes.

Acknowledgments: We are grateful to K. Teter (University of Florida) for his generous gift of the Vero-d2EGFP cell line.

Conflicts of Interest: The authors declare no conflict of interest. The funders had no role in the design of the study; in the collection, analysis, or interpretation of data; in the writing of the manuscript; or in the decision to publish the results.

\section{References}

1. Hofmann, S.L. Southwestern internal medicine conference: Shiga-like toxins in hemolytic-uremic syndrome and thrombotic thrombocytopenic purpura. Am. J. Med. Sci. 1993, 306, 398-406. [CrossRef] [PubMed]

2. Karmali, M.A.; Steele, B.T.; Petric, M.; Lim, C. Sporadic cases of haemolytic-uraemic syndrome associated with faecal cytotoxin and cytotoxin-producing Escherichia coli in stools. Lancet 1983, 1, 619-620. [CrossRef]

3. Riley, L.W.; Remis, R.S.; Helgerson, S.D.; McGee, H.B.; Wells, J.G.; Davis, B.R.; Hebert, R.J.; Olcott, E.S.; Johnson, L.M.; Hargrett, N.T.; et al. Hemorrhagic colitis associated with a rare Escherichia coli serotype. N. Engl. J. Med. 1983, 308, 681-685. [CrossRef] [PubMed]

4. Karmali, M.A.; Mascarenhas, M.; Shen, S.; Ziebell, K.; Johnson, S.; Reid-Smith, R.; Isaac-Renton, J.; Clark, C.; Rahn, K.; Kaper, J.B. Association of genomic O island 122 of Escherichia coli EDL 933 with verocytotoxin-producing Escherichia coli seropathotypes that are linked to epidemic and/or serious disease. J. Clin. Microbiol. 2003, 41, 4930-4940. [CrossRef]

5. Karmali, M.A.; Gannon, V.; Sargeant, J.M. Verocytotoxin-producing Escherichia coli (VTEC). Vet. Microbiol. 2010, 140, 360-370. [CrossRef] [PubMed]

6. Soysal, N.; Mariani-Kurkdjian, P.; Smail, Y.; Liguori, S.; Gouali, M.; Loukiadis, E.; Fach, P.; Bruyand, M.; Blanco, J.; Bidet, P.; et al. Enterohemorrhagic Escherichia coli Hybrid Pathotype O80:H2 as a New Therapeutic Challenge. Emerg. Infect. Dis. 2016, 22, 1604-1612. [CrossRef] [PubMed]

7. Valilis, E.; Ramsey, A.; Sidiq, S.; DuPont, H.L. Non-O157 Shiga toxin-producing Escherichia coli-A poorly appreciated enteric pathogen: Systematic review. Int. J. Infect. Dis. 2018, 76, 82-87. [CrossRef]

8. Endo, Y.; Tsurugi, K.; Yutsudo, T.; Takeda, Y.; Ogasawara, T.; Igarashi, K. Site of action of a Vero toxin (VT2) from Escherichia coli O157:H7 and of Shiga toxin on eukaryotic ribosomes. RNA N-glycosidase activity of the toxins. Eur. J. Biochem. 1988, 171, 45-50. [CrossRef]

9. Schuller, S. Shiga toxin interaction with human intestinal epithelium. Toxins 2011, 3, 626-639. [CrossRef]

10. Ray, P.E.; Liu, X.H. Pathogenesis of Shiga toxin-induced hemolytic uremic syndrome. Pediatr. Nephrol. 2001, 16, 823-839. [CrossRef] 
11. Melton-Celsa, A.R. Shiga toxin (Stx) classification, structure, and function. Microbiol. Spectr. $2014,2$. [CrossRef] [PubMed]

12. Kruger, A.; Lucchesi, P.M. Shiga toxins and stx phages: Highly diverse entities. Microbiology 2015, 161, 451-462. [CrossRef] [PubMed]

13. Bielaszewska, M.; Friedrich, A.W.; Aldick, T.; Schurk-Bulgrin, R.; Karch, H. Shiga toxin activatable by intestinal mucus in Escherichia coli isolated from humans: Predictor for a severe clinical outcome. Clin. Infect. Dis 2006, 43, 1160-1167. [CrossRef] [PubMed]

14. Siegler, R.L.; Obrig, T.G.; Pysher, T.J.; Tesh, V.L.; Denkers, N.D.; Taylor, F.B. Response to Shiga toxin 1 and 2 in a baboon model of hemolytic uremic syndrome. Pediatr. Nephrol. 2003, 18, 92-96. [CrossRef]

15. Schmidt, M.A. LEEways: Tales of EPEC, ATEC and EHEC. Cell. Microbiol. 2010, 12, 1544-1552. [CrossRef]

16. Kulasekara, B.R.; Jacobs, M.; Zhou, Y.; Wu, Z.; Sims, E.; Saenphimmachak, C.; Rohmer, L.; Ritchie, J.M.; Radey, M.; McKevitt, M.; et al. Analysis of the genome of the Escherichia coli O157:H7 2006 spinach-associated outbreak isolate indicates candidate genes that may enhance virulence. Infect. Immun. 2009, 77, 3713-3721. [CrossRef]

17. Manning, S.D.; Motiwala, A.S.; Springman, A.C.; Qi, W.; Lacher, D.W.; Ouellette, L.M.; Mladonicky, J.M.; Somsel, P.; Rudrik, J.T.; Dietrich, S.E.; et al. Variation in virulence among clades of Escherichia coli O157:H7 associated with disease outbreaks. Proc. Natl. Acad. Sci. USA 2008, 105, 4868-4873. [CrossRef]

18. Gardner, A.M.; Gessner, C.R.; Gardner, P.R. Regulation of the nitric oxide reduction operon (norRVW) in Escherichia coli. Role of NorR and sigma54 in the nitric oxide stress response. J. Biol. Chem. 2003, 278, 10081-10086. [CrossRef]

19. Gardner, A.M.; Helmick, R.A.; Gardner, P.R. Flavorubredoxin, an inducible catalyst for nitric oxide reduction and detoxification in Escherichia coli. J. Biol. Chem. 2002, 277, 8172-8177. [CrossRef]

20. Hutchings, M.I.; Mandhana, N.; Spiro, S. The NorR protein of Escherichia coli activates expression of the flavorubredoxin gene nor $V$ in response to reactive nitrogen species. J. Bacteriol. 2002, 184, 4640-4643. [CrossRef]

21. Bogdan, C. Nitric oxide synthase in innate and adaptive immunity: An update. Trends Immunol. 2015, 36, 161-178. [CrossRef] [PubMed]

22. Branchu, P.; Matrat, S.; Vareille, M.; Garrivier, A.; Durand, A.; Crepin, S.; Harel, J.; Jubelin, G.; Gobert, A.P. NsrR, GadE, and GadX interplay in repressing expression of the Escherichia coli O157:H7 LEE pathogenicity island in response to nitric oxide. PLoS Pathog. 2014, 10, e1003874. [CrossRef] [PubMed]

23. Gobert, A.P.; Vareille, M.; Glasser, A.L.; Hindre, T.; de Sablet, T.; Martin, C. Shiga toxin produced by enterohemorrhagic Escherichia coli inhibits PI3K/NF-kappaB signaling pathway in globotriaosylceramide-3-negative human intestinal epithelial cells. J. Immunol. 2007, 178, 8168-8174. [CrossRef] [PubMed]

24. Ichimura, K.; Shimizu, T.; Matsumoto, A.; Hirai, S.; Yokoyama, E.; Takeuchi, H.; Yahiro, K.; Noda, M. Nitric oxide-enhanced Shiga toxin production was regulated by Fur and RecA in enterohemorrhagic Escherichia coli O157. Microbiologyopen 2017, 6. [CrossRef]

25. Naili, I.; Gardette, M.; Garrivier, A.; Daniel, J.; Desvaux, M.; Pizza, M.; Gobert, A.; Marchal, T.; Loukiadis, E.; Jubelin, G. Interplay between enterohaemorrhagic Escherichia coli and nitric oxide during the infectious process. Emerg. Microbes Infect. 2020, 9, 1065-1076. [CrossRef]

26. Shimizu, T.; Tsutsuki, H.; Matsumoto, A.; Nakaya, H.; Noda, M. The nitric oxide reductase of enterohaemorrhagic Escherichia coli plays an important role for the survival within macrophages. Mol. Microbiol. 2012, 85, 492-512. [CrossRef]

27. Espie, E.; Vaillant, V.; Mariani-Kurkdjian, P.; Grimont, F.; Martin-Schaller, R.; De Valk, H.; Vernozy-Rozand, C. Escherichia coli $\mathrm{O} 157$ outbreak associated with fresh unpasteurized goats' cheese. Epidemiol. Infect. 2006, 134, 143-146. [CrossRef]

28. Douellou, T.; Delannoy, S.; Ganet, S.; Mariani-Kurkdjian, P.; Fach, P.; Loukiadis, E.; Montel, M.; Thevenot-Sergentet, D. Shiga toxin-producing Escherichia coli strains isolated from dairy products-Genetic diversity and virulence gene profiles. Int. J. Food Microbiol. 2016, 232, 52-62. [CrossRef]

29. King, L.A.; Loukiadis, E.; Mariani-Kurkdjian, P.; Haeghebaert, S.; Weill, F.X.; Baliere, C.; Ganet, S.; Gouali, M.; Vaillant, V.; Pihier, N.; et al. Foodborne transmission of sorbitol-fermenting Escherichia coli O157:H7 via ground beef: An outbreak in northern France, 2011. Clin. Microbiol. Infect. 2014, 20, O1136-O1144. [CrossRef] 
30. Kerangart, S.; Douellou, T.; Delannoy, S.; Fach, P.; Beutin, L.; Sergentet-Thevenot, D.; Cournoyer, B.; Loukiadis, E. Variable tellurite resistance profiles of clinically-relevant Shiga toxin-producing Escherichia coli (STEC) influence their recovery from foodstuffs. Food Microbiol. 2016, 59, 32-42. [CrossRef]

31. Bonanno, L.; Loukiadis, E.; Mariani-Kurkdjian, P.; Oswald, E.; Garnier, L.; Michel, V.; Auvray, F. Diversity of Shiga Toxin-Producing Escherichia coli (STEC) O26:H11 Strains Examined via stx Subtypes and Insertion Sites of Stx and EspK Bacteriophages. Appl. Environ. Microbiol. 2015, 81, 3712-3721. [CrossRef] [PubMed]

32. Teel, L.D.; Melton-Celsa, A.R.; Schmitt, C.K.; O'Brien, A.D. One of two copies of the gene for the activatable shiga toxin type 2d in Escherichia coli O91:H21 strain B2F1 is associated with an inducible bacteriophage. Infect. Immun. 2002, 70, 4282-4291. [CrossRef] [PubMed]

33. Ito, H.; Terai, A.; Kurazono, H.; Takeda, Y.; Nishibuchi, M. Cloning and nucleotide sequencing of Vero toxin 2 variant genes from Escherichia coli O91:H21 isolated from a patient with the hemolytic uremic syndrome. Microb. Pathog. 1990, 8, 47-60. [CrossRef]

34. Galia, W.; Mariani-Kurkdjian, P.; Loukiadis, E.; Blanquet-Diot, S.; Leriche, F.; Brugere, H.; Shima, A.; Oswald, E.; Cournoyer, B.; Thevenot-Sergentet, D. Genome sequence and annotation of a human infection isolate of Escherichia coli O26:H11 involved in a raw milk cheese outbreak. Genome Announc. 2015, 3. [CrossRef]

35. Lindgren, S.W.; Melton, A.R.; O’Brien, A.D. Virulence of enterohemorrhagic Escherichia coli O91:H21 clinical isolates in an orally infected mouse model. Infect. Immun. 1993, 61, 3832-3842. [CrossRef] [PubMed]

36. Quinones, B.; Massey, S.; Friedman, M.; Swimley, M.S.; Teter, K. Novel cell-based method to detect Shiga toxin 2 from Escherichia coli O157:H7 and inhibitors of toxin activity. Appl. Environ. Microbiol. 2009, 75, 1410-1416. [CrossRef]

37. Gardette, M.; Le Hello, S.; Mariani-Kurkdjian, P.; Fabre, L.; Gravey, F.; Garrivier, A.; Loukiadis, E.; Jubelin, G. Identification and prevalence of in vivo-induced genes in enterohaemorrhagic Escherichia coli. Virulence 2019, 10, 180-193. [CrossRef] [PubMed]

38. John, M.; Kudva, I.T.; Griffin, R.W.; Dodson, A.W.; McManus, B.; Krastins, B.; Sarracino, D.; Progulske-Fox, A.; Hillman, J.D.; Handfield, M.; et al. Use of in vivo-induced antigen technology for identification of Escherichia coli O157:H7 proteins expressed during human infection. Infect. Immun. 2005, 73, 2665-2679. [CrossRef]

39. Vareille, M.; de Sablet, T.; Hindre, T.; Martin, C.; Gobert, A.P. Nitric oxide inhibits Shiga-toxin synthesis by enterohemorrhagic Escherichia coli. Proc. Natl. Acad. Sci. USA 2007, 104, 10199-10204. [CrossRef]

40. Hausladen, A.; Gow, A.; Stamler, J.S. Flavohemoglobin denitrosylase catalyzes the reaction of a nitroxyl equivalent with molecular oxygen. Proc. Natl. Acad. Sci. USA 2001, 98, 10108-10112. [CrossRef]

41. Poock, S.R.; Leach, E.R.; Moir, J.W.; Cole, J.A.; Richardson, D.J. Respiratory detoxification of nitric oxide by the cytochrome c nitrite reductase of Escherichia coli. J. Biol. Chem. 2002, 277, 23664-23669. [CrossRef] [PubMed]

42. Wang, J.; Vine, C.E.; Balasiny, B.K.; Rizk, J.; Bradley, C.L.; Tinajero-Trejo, M.; Poole, R.K.; Bergaust, L.L.; Bakken, L.R.; Cole, J.A. The roles of the hybrid cluster protein, Hcp and its reductase, Hcr, in high affinity nitric oxide reduction that protects anaerobic cultures of Escherichia coli against nitrosative stress. Mol. Microbiol. 2016, 100, 877-892. [CrossRef] [PubMed]

43. Justino, M.C.; Vicente, J.B.; Teixeira, M.; Saraiva, L.M. New genes implicated in the protection of anaerobically grown Escherichia coli against nitric oxide. J. Biol. Chem. 2005, 280, 2636-2643. [CrossRef] [PubMed]

44. Robinson, J.L.; Brynildsen, M.P. Discovery and dissection of metabolic oscillations in the microaerobic nitric oxide response network of Escherichia coli. Proc. Natl. Acad. Sci. USA 2016, 113, E1757-E1766. [CrossRef] [PubMed]

45. Shimizu, T.; Matsumoto, A.; Noda, M. Cooperative roles of nitric oxide-metabolizing enzymes to counteract nitrosative stress in enterohemorrhagic Escherichia coli. Infect. Immun. 2019, 87. [CrossRef] [PubMed]

46. Mehta, H.H.; Liu, Y.; Zhang, M.Q.; Spiro, S. Genome-wide analysis of the response to nitric oxide in uropathogenic Escherichia coli CFT073. Microbial Genom. 2015, 1, e000031. [CrossRef]

47. Robinson, C.M.; Sinclair, J.F.; Smith, M.J.; O’Brien, A.D. Shiga toxin of enterohemorrhagic Escherichia coli type O157:H7 promotes intestinal colonization. Proc. Natl. Acad. Sci. USA 2006, 103, 9667-9672. [CrossRef]

48. Liu, B.; Yin, X.; Feng, Y.; Chambers, J.R.; Guo, A.; Gong, J.; Zhu, J.; Gyles, C.L. Verotoxin 2 enhances adherence of enterohemorrhagic Escherichia coli O157:H7 to intestinal epithelial cells and expression of $\beta 1$-integrin by IPEC-J2 cells. Appl. Environ. Microbiol. 2010, 76, 4461-4468. [CrossRef] 
49. Bang, I.S.; Liu, L.; Vazquez-Torres, A.; Crouch, M.L.; Stamler, J.S.; Fang, F.C. Maintenance of nitric oxide and redox homeostasis by the Salmonella flavohemoglobin hmp. J. Biol. Chem. 2006, 281, 28039-28047. [CrossRef]

50. Jones-Carson, J.; Husain, M.; Liu, L.; Orlicky, D.J.; Vazquez-Torres, A. Cytochrome bd-dependent bioenergetics and antinitrosative defenses in Salmonella Pathogenesis. mBio 2016, 7. [CrossRef]

51. Karlinsey, J.E.; Bang, I.S.; Becker, L.A.; Frawley, E.R.; Porwollik, S.; Robbins, H.F.; Thomas, V.C.; Urbano, R.; McClelland, M.; Fang, F.C. The NsrR regulon in nitrosative stress resistance of Salmonella enterica serovar Typhimurium. Mol. Microbiol. 2012, 85, 1179-1193. [CrossRef] [PubMed]

52. Richardson, A.R.; Dunman, P.M.; Fang, F.C. The nitrosative stress response of Staphylococcus aureus is required for resistance to innate immunity. Mol. Microbiol. 2006, 61, 927-939. [CrossRef] [PubMed]

53. Sebbane, F.; Lemaitre, N.; Sturdevant, D.E.; Rebeil, R.; Virtaneva, K.; Porcella, S.F.; Hinnebusch, B.J. Adaptive response of Yersinia pestis to extracellular effectors of innate immunity during bubonic plague. Proc. Natl. Acad. Sci. USA 2006, 103, 11766-11771. [CrossRef] [PubMed]

54. Svensson, L.; Poljakovic, M.; Save, S.; Gilberthorpe, N.; Schon, T.; Strid, S.; Corker, H.; Poole, R.K.; Persson, K. Role of flavohemoglobin in combating nitrosative stress in uropathogenic Escherichia coli: Implications for urinary tract infection. Microb. Pathog. 2010, 49, 59-66. [CrossRef] [PubMed]

55. Arai, H.; Iiyama, K. Role of nitric oxide-detoxifying enzymes in the virulence of Pseudomonas aeruginosa against the silkworm, Bombyx mori. Biosci. Biotechnol. Biochem. 2013, 77, 198-200. [CrossRef] [PubMed]

56. Liu, J.; Dong, Y.; Wang, N.; Ma, S.; Lu, C.; Liu, Y. Diverse effects of nitric oxide reductase NorV on Aeromonas hydrophila virulence-associated traits under aerobic and anaerobic conditions. Vet. Res. 2019, 50, 67. [CrossRef]

57. Burton, N.A.; Schurmann, N.; Casse, O.; Steeb, A.K.; Claudi, B.; Zankl, J.; Schmidt, A.; Bumann, D. Disparate impact of oxidative host defenses determines the fate of Salmonella during systemic infection in mice. Cell Host Microbe 2014, 15, 72-83. [CrossRef]

58. Shimizu, T.; Hirai, S.; Yokoyama, E.; Ichimura, K.; Noda, M. An evolutionary analysis of nitric oxide reductase gene norV in enterohemorrhagic Escherichia coli O157. Infect. Genet. Evol. 2015, 33, 176-181. [CrossRef]

59. Boerlin, P.; McEwen, S.A.; Boerlin-Petzold, F.; Wilson, J.B.; Johnson, R.P.; Gyles, C.L. Associations between virulence factors of Shiga toxin-producing Escherichia coli and disease in humans. J. Clin. Microbiol. 1999, 37, 497-503. [CrossRef]

60. Ostroff, S.M.; Tarr, P.I.; Neill, M.A.; Lewis, J.H.; Hargrett-Bean, N.; Kobayashi, J.M. Toxin genotypes and plasmid profiles as determinants of systemic sequelae in Escherichia coli O157:H7 infections. J. Infect. Dis. 1989, 160, 994-998. [CrossRef]

61. Gill, A.E.; Amyes, S.G. The contribution of a novel ribosomal S12 mutation to aminoglycoside resistance of Escherichia coli mutants. J. Chemother. 2004, 16, 347-349. [CrossRef] [PubMed]

62. Datsenko, K.A.; Wanner, B.L. One-step inactivation of chromosomal genes in Escherichia coli K-12 using PCR products. Proc. Natl. Acad. Sci. USA 2000, 97, 6640-6645. [CrossRef] [PubMed]

63. Quinones, B.; Swimley, M.S. Use of a Vero cell-based fluorescent assay to assess relative toxicities of Shiga toxin 2 subtypes from Escherichia coli. Methods Mol. Biol. 2011, 739, 61-71. [CrossRef] [PubMed]

64. King, L.A.; Mailles, A.; Mariani-Kurkdjian, P.; Vernozy-Rozand, C.; Montet, M.P.; Grimont, F.; Pihier, N.; Devalk, H.; Perret, F.; Bingen, E.; et al. Community-wide outbreak of Escherichia coli O157:H7 associated with consumption of frozen beef burgers. Epidemiol. Infect. 2009, 137, 889-896. [CrossRef] [PubMed]

65. Hayashi, T.; Makino, K.; Ohnishi, M.; Kurokawa, K.; Ishii, K.; Yokoyama, K.; Han, C.G.; Ohtsubo, E.; Nakayama, K.; Murata, T.; et al. Complete Genome Sequence of Enterohemorrhagic Escherichia coli O157:H7 and Genomic Comparison with a Laboratory Strain K-12. DNA Res. 2001, 8, 11-22. [CrossRef] [PubMed]

66. Neto, M.; Delannoy, S.; Auvray, F.; Oswald, E.; Fach, P.; Loukiadis, E. Public health significance of E. coli 026 isolated from foodstuffs: Toward genetic predictors of their virulence. Zoonoses Public Health 2012, 59, $19-90$. [CrossRef]

67. Ogura, Y.; Gotoh, Y.; Itoh, T.; Sato, M.P.; Seto, K.; Yoshino, S.; Isobe, J.; Etoh, Y.; Kurogi, M.; Kimata, K.; et al. Population structure of Escherichia coli O26: $\mathrm{H} 11$ with recent and repeated stx2 acquisition in multiple lineages. Microb. Genom. 2017, 3, e000141. [CrossRef]

68. Mariani-Kurkdjian, P.; Denamur, E.; Milon, A.; Picard, B.; Cave, H.; Lambert-Zechovsky, N.; Loirat, C.; Goullet, P.; Sansonetti, P.J.; Elion, J. Identification of a Clone of Escherichia coli 0103:H2 as a Potential Agent of Hemolytic-Uremic Syndrome in France. J. Clin. Microbiol. 1993, 31, 296-301. [CrossRef] 
69. Zhang, W.L.; Köhler, B.; Oswald, E.; Beutin, L.; Karch, H.; Morabito, S.; Caprioli, A.; Suerbaum, S.; Schmidt, H. Genetic Diversity of Intimin Genes of Attaching and Effacing Escherichia coli Strains. J. Clin. Microbiol. 2002, 40, 4486-4492. [CrossRef]

70. Bibbal, D.; Loukiadis, E.; Kérourédan, M.; Ferré, F.; Dilasser, F.; Peytavin De Garam, C.; Cartier, P.; Oswald, E.; Gay, E.; Auvray, F.; et al. Prevalence of Carriage of Shiga Toxin-Producing Escherichia coli Serotypes O157:H7, O26:H11, O103:H2, O111:H8, and O145:H28 among Slaughtered Adult Cattle in France. Appl. Environ. Microbiol. 2015, 81, 1397-1405. [CrossRef]

(C) 2020 by the authors. Licensee MDPI, Basel, Switzerland. This article is an open access article distributed under the terms and conditions of the Creative Commons Attribution (CC BY) license (http://creativecommons.org/licenses/by/4.0/). 